\title{
Gravitational Waves from Rotating Neutron Stars and Evaluation of Fast Chirp Transform Techniques
}

\author{
Tod E. Strohmayer $\dagger \S$ \\ $\dagger$ Laboratory for High Energy Astrohphysics, NASA/GSFC, Greenbelt, MD, \\ 20771
}

\begin{abstract}
.
$\mathrm{X}$-ray observations suggest that neutron stars in low mass X-ray binaries (LMXB) are rotating with frequencies from $300-600 \mathrm{~Hz}$. These spin rates are significantly less than the break-up rates for essentially all realistic neutron star equations of state, suggesting that some process may limit the spin frequencies of accreting neutron stars to this range. If the accretion induced spin up torque is in equilibrium with gravitational radiation losses, these objects could be interesting sources of gravitational waves. I present a brief summary of current measurements of neutron star spins in LMXBs based on the observations of high-Q oscillations during thermonuclear bursts (so called "burst oscillations"). Further measurements of neutron star spins will be important in exploring the gravitational radiation hypothesis in more detail. To this end I also present a study of fast chirp transform (FCT) techniques as described by Jenet \& Prince $(2000)$ in the context of searching for the chirping signals observed during $\mathrm{X}$-ray bursts.
\end{abstract}

\section{Introduction}

$\mathrm{X}$-ray binaries are potentially among the most interesting sources of gravitational wave emission which current and future gravitational wave detectors will attempt to study. The high frequency signal produced during binary inspiral and ring down of black hole and neutron star binaries contains detailed information on the properties of the compact object as well as the structure of spacetime in its vicinity. These objects will be prime targets for ground based detectors such as LIGO which because of seismic noise are only sensitive in the high frequency range above $\sim 100 \mathrm{~Hz}$.

Neutron stars are compelling targets of investigation because of the extreme physical conditions which exist in their interiors and immediate environs. For example, the signals produced by inspiral of a neutron star binary just before merger depend on the equation of state (EOS) at supranuclear density, a quantity which is still not precisely constrained by currently available astrophysics and nuclear physics data (see for example Heiselberg \& Hjorth-Jensen 1999). Moreover, fundamental properties of the star, such as its mass, can be extracted if the gravitational wave signal can be measured. Thus gravitational wave astronomy can in principle provide new probes of fundamental physics as well as advancing neutron star astrophysics.

$\S$ email: stroh@clarence.gsfc.nasa.gov 
Table 1. Burst Oscillation Sources and Properties

\begin{tabular}{cccc}
\hline Sources & Frequency $(\mathrm{Hz})$ & $\Delta \nu(\mathrm{kHz}$ QPO, in Hz) & References $^{1}$ \\
\hline $4 \mathrm{U} \mathrm{1728-34}$ & 363 & $363-280$ & $1,2,3,4,5,13,14$ \\
$4 \mathrm{U} 1636-53$ & 290,580 & 251 & 6,7 \\
$4 \mathrm{U} \mathrm{1702-429}$ & 330 & $315-344$ & 4,9 \\
KS 1731-260 & 524 & 260 & $10,11,12$ \\
Galactic Center & 589 & Unknown & 15 \\
Aql X-1 & 549 & Unknown & 16,17 \\
X1658-298 & 567 & Unknown & 18 \\
4U 1916-053 & 270 & $290-348$ & 19,20 \\
$4 U 1608-52$ & 619 & $225-325$ & 8,21 \\
SAX J1808-369 & 401 & Unknown & 22,23 \\
\hline
\end{tabular}

${ }^{1}$ References: (1) Strohmayer et al. (1996); (2) Strohmayer, Zhang, \& Swank (1997); (3) Mendez \& van der Klis (1999); (4) Strohmayer \& Markwardt (1999); (5) Strohmayer et al. (1998b); (6) Strohmayer et al. (1998a); (7) Miller (1999); (8) Mendez et al. (1998); (9) Markwardt, Strohmayer \& Swank (1999) (10) Smith, Morgan, \& Bradt (1997); (11) Wijnands \& van der Klis (1998); (12) Muno et al. (2000); (13) van Straaten et al. (2000); (14) Franco (2000); (15) Strohmayer et al (1997); (16) Zhang et al. (1998); (17) Ford (1999); (18) Wijnands, Strohmayer \& Franco (2000); (19) Boirin et al. (2000); (20) Galloway et al. $(2000) ;(21)$ Chakrabarty $(2000) ;(22)$ Heise (2000); (23) Ford (2000)

\section{Burst Oscillations}

Radio observations provided the first indications that some neutron stars are spinning with periods approaching $1.5 \mathrm{~ms}$ (Backer et al. 1982). These rapidly rotating neutron stars are observed as either isolated or binary radio pulsars. Binary evolution models indicate that neutron stars accreting mass from a companion can be spun up, or "recycled", to millisecond periods (see for example Webbink, Rappaport \& Savonije 1983). This formation mechanism likely accounts for a substantial fraction of the observed population of millisecond radio pulsars, however, other formation scenarios have also been proposed (see van den Heuvel \& Bitzaraki 1995; van Paradijs et al. 1997).

In recent years direct evidence linking the formation of rapidly rotating neutron stars to accreting X-ray binaries has been provided by data from the Rossi Xray Timing Explorer (RXTE). The first evidence came from the discovery of high amplitude, nearly coherent X-ray brightness oscillations (so called "burst oscillations") during thermonuclear flashes from several neutron star binaries (see Strohmayer 2000 for a recent review). These oscillations likely result from spin modulation of either one or a pair of antipodal "hot spots" generated as a result of the thermonuclear burning of matter accreted on the neutron star surface. Indisputable evidence that neutron stars in X-ray binaries can indeed be rotating rapidly then came with the discovery of the first accreting millisecond X-ray pulsar SAX J1808-369 (Wijnands \& van der Klis 1998; Chakrabarty \& Morgan 1998), which is spinning at $401 \mathrm{~Hz}$.

The observed distribution of burst oscillation frequencies, including the $401 \mathrm{~Hz}$ pulsar, is very similar to the observed distribution of millisecond radio pulsars. Table 1 lists properties of burst oscillations in the sources in which they have been detected. The RXTE observations suggest that the spin frequencies of neutron stars in accreting 


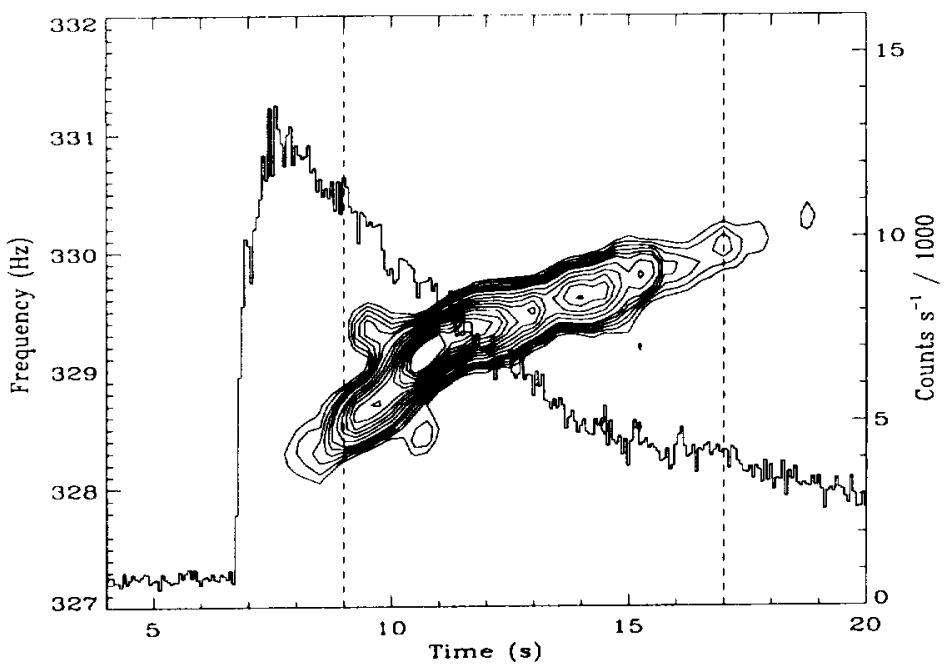

Figure 1. Contour plot of the dynamic power spectrum of a burst from the LMXB 4U 1702-429 observed on April 1, 2001 (UTC) with the PCA onboard RXTE. Shown are contours of constant power spectral amplitude as a function of frequency and time. The burst profile is also plotted (right axis). The oscillation frequency increase from about 328 to $330 \mathrm{~Hz}$ in $\sim 10$ seconds.

binaries span a relatively narrow range from $300-600 \mathrm{~Hz}$. Moreover, these observed frequencies are significantly less than the maximum neutron star spin rates for almost all but the stiffest neutron star equations of state (Cook, Shapiro \& Teukolsky 1994). In principle, accretion should drive the spin frequencies close to break-up, unless some other mechanism intervenes to remove the accreted angular momentum.

\section{Gravitational Wave Emission from Rotating Neutron Stars}

Bildsten (1998) recently proposed that the angular momentum gain from accretion could be offset by gravitational radiation losses if a misaligned quadrupole moment of order $10^{-8}-10^{-7} I_{N S}$ could be sustained in the neutron star crust. Here $I_{N S}$ is the moment of inertia of the neutron star. In this scenario the strong spin frequency dependence of gravitational radiation losses sets the limit on the observed spin frequencies. Recent theoretical work has also shown that an $r$-mode instability in rotating neutron stars may also be important in limiting the spins of neutron stars via gravitational wave emission (see Bildsten 1998; Andersson, Kokkotas, \& Stergioulas 1999; Levin 1999; Ushomirsky, Bildsten \& Cutler 2000; Andersson et al. 2000).

The strong $\nu^{6}$ frequency dependence of the energy radiated by gravitational waves means that rapidly rotating neutron stars with misaligned quadrupole moments might have observationally interesting gravitational wave amplitudes. The spin periods of neutron stars inferred from burst oscillations cluster rather tightly in the range from $300-600 \mathrm{~Hz}$. White \& Zhang (1997) suggested that the observed range of spin frequencies could be produced if these neutron stars were spinning in magnetic equilibrium. However, in order for the observed frequencies to be similar would require that mass accretion rate and the magnetic moment be correlated. It is not presently 
known if such a correlation is to be expected based on theoretical grounds. The model proposed by Bildsten (1998) suggests that the spins of these neutron stars may be limited by the emission of gravitational waves (see also Wagoner 1984). The spin down torque due to gravitational wave emission is proportional to $\nu^{5}$ so that one would expect a critical spin frequency above which accretion torques are cancelled out by gravitational wave losses. By equating the characteristic accretion torque with the gravitational wave torque one can determine the average quadrupole moment required to maintain the critical frequency. For the mass accretion rates characteristic of LMXBs and a critical spin frequency of $300 \mathrm{~Hz}$ one obtains a quadrupole moment $Q \sim 4.5 \times 10^{37} \mathrm{gm} \mathrm{cm}{ }^{2}$, or about $5 \times 10^{-8} I_{N S}$ (see Bildsten 1998). The question that remains is whether or not such a quadrupole moment can be routinely generated within a neutron star. This is presently an area of active research (see for example, Ushomirsky, Bildsten \& Cutler 2000).

Regardless of the mechanism, if the accretion torque is indeed balanced by gravitational wave losses then the amplitude of the gravitational radiation can be calculated (see Wagoner 1984; Bildsten 1998). The dimensionless strain $h$ is in the range from $h \sim 10^{-27}-10^{-26}$. Although less than the estimated sensitivity for LIGO I, one can greatly improve the sensitivity by pulse folding if the rotational ephemeris of the neutron star is known (see Brady \& Creighton 1999; Ushomirsky, Bildsten \& Cutler 2000). Current estimates indicate that a narrow band configuration for LIGO-II will reach interesting search limits for these neutron stars, especially for the brightest of the LMXBs, for example, Sco X-1 (Ushomirsky, Bildsten \& Cutler 2000). This also provides strong motivation for additional deep X-ray timing searches in order to detect coherent pulsations in more LMXBs and to measure the pulse ephemerides so as to improve searches for gravitational wave emission. It also illustrates the strong synergism between X-ray and gravitational wave astronomy in the context of neutron star binaries.

\section{Searches for Chirping Signals: Burst Oscillations}

One way to search for additional neutron star spin periods is to try and make more sensitive searches for burst oscillations. Since the signals during many bursts are known to increase with time (ie. they are chirps, see Figure 1), it is likely that more general search techniques which allow for frequency changes will be better at detecting weak burst oscillation signals. Prince \& Jenet (2000) have recently described a general method based on multidimensional FFTs to search for signals with varying frequency. They call this technique the Fast Chirp Transform (FCT). In an effort to develop more sensitive searches for burst oscillations as well as techniques to search for GW emission from rotating neutron stars I have begun evaluating the FCT on X-ray burst oscillation data which are known to contain chirping signals. Here we briefly describe the simplest 2-parameter (linear) chirp and the corresponding 2-d FCT.

A signal with a linearly increasing frequency is of the form $h_{s}(t)=A(t) \sin (2 \pi(\nu t+$ $\left.\left.\dot{\nu} t^{2}\right)\right)$, that is the phase advance is quadratic in time. The FCT is an approximation to the exact Fourier transform for such a signal; $H_{k_{0}, k_{1}}=\sum_{j_{0}} h_{j_{0}} \exp \left(2 \pi i\left(k_{0}\left(j_{0} / N_{0}\right)+\right.\right.$ $\left.\left.k_{1}\left(j_{0} / N_{0}\right)^{2}\right)\right)$. The second term in the exponential represents the quadratic phase advance produced by the changing frequency. The sum is over $j_{0}$ with $0<j_{0}<N_{0}-1$. That is, the data, $h_{j_{0}}$, are a discretely sampled time series with $N_{0}$ bins. The FCT approximates this expression by breaking up the single sum into a double sum by defining a set of $N_{1}$ subintervals of the data. Within each subinterval the phase 
advance produced by the quadratic term is required to have the same value, that is, it increments uniformly as a "second index", and the approximate expression can be evaluated as a 2-d FFT. This requires only $O\left(N_{0} N_{1} \log _{2}\left(N_{0} N_{1}\right)\right)$ operations as opposed to $O\left(N_{0}^{2}\right)$, and is therefore much more computationally efficient. There is a simple geometrical way to see how the 2-d FFT implementation of the FCT is constructed. One forms a two dimensional array, one dimension being $N_{0}$ in size (the number of samples in the original time series), and the other being of length $N_{1}$ (the number of subintervals). The length of each of the $N_{1}$ data segments packed along the $N_{0}$ component is determined by the phase evolution function. Since the increase in phase is quadratic in time, subsequent data segments down the $N_{1}$ direction become shorter because they must represent the same total phase advance. In this way the boundaries of the subintervals are specified uniquely by the phase evolution function (see Prince \& Jenet 2000).

The range of $\dot{\nu}$ searched is determined by $N_{1}$, the number of subintervals of the original time series. The maximum $\dot{\nu}$ searched is simply $\dot{\nu}_{\max }=N_{1} / 2 T^{2}$, where $T$ is the length of the original time series. Both positive and negative values are searched, analogously to the positive and negative frequencies of a standard FFT power spectrum, in this case, however, the FCT is not in general symmetric with regard to positive or negative $\dot{\nu}$.

I have implemented the 2-d FCT in IDL using the formalism outlined in Jenet \& Prince (2000). I have evaluated the FCT by looking at burst oscillation data in bursts with detected oscillations from the LMXB 4U 1702-429 (see Markwardt, Strohmayer \& Swank 1998). Figure 2 shows a comparison between a standard FFT power spectral analysis and one using the FCT for the burst shown in Figure 1. As can be seen in Figure 1, this burst has a strong oscillation at $\sim 330 \mathrm{~Hz}$. I used 8 seconds of data sampled at $4096 \mathrm{~Hz}$ (the interval between the dashed vertical lines in Figure 1). The data were first mixed with a $f_{m i x}=326 \mathrm{~Hz}$ modulation in order to bring the oscillation signal down to lower frequency, and therefore reduce $N_{0}$. This is similar to a heterodyne procedure. Figure 2 (left) shows a plot of the peak power in the FCT as a function of $\dot{\nu}$. One can see that the signal strength is greatly increased at $\dot{\nu} \sim 0.075 \mathrm{~s}^{-2}$ compared with $\dot{\nu}=0$. In Figure 2 (right) the power spectrum with the highest value of the FCT power is compared with that computed with $\dot{\nu}=0$ in Figure 2 (right). The FCT at positive $\dot{\nu}$ recovers a narrow, coherent peak whereas that computed with no frequency derivative is smeared out and weaker. This demonstrates the effectiveness of the FCT for detecting these kinds of signals. A detailed search for burst oscillations in new data will be presented elsewhere.

\section{Acknowledgments}

I thank Tom Prince for many helpful discussions and suggestions.

\section{References}

Andersson, N. Jones, D. I., Kokkotas, K. D. \& Stergioulas, N. 2000, ApJ, 534, L75

Andersson, N. Kokkotas, K. D. \& Stergioulas, N. 1999, ApJ, 516, 307

Backer, D. C., Kulkarni, S. R., Heiles, C., Davis, M. M. \& Goss, W. M. 1982, Nature, 300, 615

Bildsten, L. 1998, ApJ, 501, L89

Boirin, L., et al. 2000, A\& A, 361,121

Brady, P. \& Creighton, T. 1999, Phys. Rev. D, in press (gr-qc/9812014)

Chakrabarty, D. 2000, Talk presented at AAS HEAD meeting, Honolulu, HI 



Figure 2. Fast chirp transform (FCT) analysis of a burst from 4 U 1702-429. The left panel shows the peak FCT power as a funtion of frequency derivative, $\dot{\nu}$. The right panel compares the FCT power spectra at two different values of $\dot{\nu}, \dot{\nu}=0$ (dashed) and $\dot{\nu}=0.078$ (solid).

Chakrabarty, D. \& Morgan, E. H. 1998, Nature, 394, 346

Ford, E. C. 2000, ApJ, 535, L119

Ford, E. C. 1999, ApJ, 519, L73

Franco, L. 2000, ApJ, submitted (astro-ph/0009189)

Galloway et al. 2000, ApJ, submitted (astro-ph/0010072)

Heise, J. et al. 2000, Talk presented at AAS HEAD meeting, Honolulu, HI

Heiselberg, H. \& Hjorth-Jensen, M. 1999, ApJ, 525, L45

Levin, Y. 1999, ApJ, 517, 328

Markwardt, C. B., Strohmayer, T. E., \& Swank, J. H. 1999, ApJ, 512, L125

Mendez, M. \& van der Klis, M. 1999, ApJ, 517, L51

Mendez, M., van der Klis, M. \& van Paradijs, J. 1998, ApJ, 506, L117

Miller, M. C. 1999, ApJ, 515, L77

Muno, M. P., Fox, D. W., Morgan, E. H. \& Bildsten, L. 2000, ApJ, 542, 1016

Prince, T. A. \& Jenet, F. A. 2000, Phys. Rev. D, 62, 122001

Smith, D., Morgan, E. H. \& Bradt, H. V. 1997, ApJ, 479, L137

Strohmayer, T. E. et al. 1996, ApJ, 469, L9

Strohmayer, T. E., Zhang, W. \& Swank, J. H. 1997, ApJ, 487, L77

Strohmayer, T. E., Jahoda, K., Giles, A. B. \& Lee, U. 1997, ApJ, 486, 355

Strohmayer, T. E. \& Markwardt, C. B. 1999, ApJ, 516, L81

Strohmayer, T. E., Zhang, W., Swank, J. H. \& Lapidus, I. 1998b, ApJ, 503, L147

Strohmayer, T. E., Zhang, W., Swank, J. H., White, N. E. \& Lapidus, I. 1998a, ApJ, 498, L135

Strohmayer, T. E. 2000, in Proceedings of X-ray Astronomy '99, Stellar Endpoints, AGN and the Diffuse X-ray Background. Bologna, Italy, (astro-ph/9911338)

Ushomirsky, G., Bildsten, L. \& Cutler, C. 2000, in 3rd Edoardo Amaldi Conference on Gravitational Waves, (astro-ph/0001129)

van den Heuvel, E. P. J. \& Bitzaraki, O. 1995, A\& A, 297, L41

van Paradijs, J., van den Heuvel, E. P. J., Kouveliotou, C., Fishman, G. J., Finger, M. H. \& Lewin, W. H. G. 1997, A\& A, 317, L9

van Straaten, S. et al. 2000, ApJ, submitted, (astro-ph/0009194)

Wagoner, R. V. 1984, ApJ, 278, 345

Webbink, R. F., Rappaport, S. A. \& Savonije, G. J. 1983, ApJ, 270, 678

White, N. E. \& Zhang, W. 1997, ApJ, 490, L87

Wijnands, R. \& van der Klis, M. 1998, Nature, 394, 344

Wijnands, R. Strohmayer, T. E. \& Franco, L. M. 2000, ApJ, in press (astro-ph/0008526)

Zhang, W. et al. 1998, ApJ, 495, L9-12 